\title{
On the effects of storytelling on wine price
}

\author{
S. Antonio ${ }^{1}$, R. Basiricò ${ }^{1}$, and A. Seccia ${ }^{2}$ \\ ${ }^{1}$ University of Foggia, Department of Sciences of Agriculture, Food and Environment, via Napoli 25, 71122 Foggia, Italy \\ ${ }^{2}$ University of Foggia, Department of Humanities, via Arpi 176, 71121 Foggia, Italy
}

\begin{abstract}
Thanks to the new developments of internet technology and social media, communication is giving consumer newfound awareness. Additionally, thanks to the technological development, the modern consumer is getting always more informed and interactive, both with other consumers and brands. He requires traditional products to be experience-based, with strong links to local culture and tradition. Storytelling based marketing is having strong impact on brand loyalty and emotional connections of consumers to wine. The study presents a review of the literature on food\&wine storytelling and social media use, and draws hypotheses to the study of the wine marketing for the future.
\end{abstract}

\section{Introduction}

Nowadays, the growing globalisation of markets has brought to an increase of the price-driven competition in the agri-food sector. At the meantime, both internet and social media marketing are growing fast, and they are replacing traditional forms of marketing and advertising, like magazines or newspapers. Communication is being miniaturized and technology is giving consumer newfound awareness. Additionally, thanks to the technological development, the modern consumer is getting always more informed and interactive, both with other consumers and brands. He requires traditional products to be experiencebased, with strong links to local culture and tradition. So, a new concept has come up, that of an "experience" product [1].

\section{Objective}

In order to differentiate and ask for a premium price, producers of quality wine are often adopting strategies aimed at identifying and translate into marketing strategies the inimitable characteristics of their products or of their winery. Storytelling techniques are nowadays widely diffused approaches at this scope. Given such a premise, the main objective of the paper is to introduce the readers to the current discussion about storytelling and wine by presenting some data and the literature, although rather limited, on the topic. The paper is structured as follows: third paragraph introduce the reader to the concept of storytelling, by discussing its definitions and theory; fourth paragraph discusses the literature on storytelling in food and wine across the world; fifth paragraph gives a brief outlook on consumers' preference with a special focus on the importance of territory for "telling good stories"; sixth paragraph illustrates the social media integration into wine industry, and connects storytelling with social media evolution. Finally concluding considerations are drawn in the seventh and last paragraph.

\section{Storytelling: Definition and theory}

"Narrative is a species-typical, reliably developing, complex cognitive process whose design is unlikely to have emerged by chance" [2]. Therefore, storytelling may be defined as the human cognitive ability to tell stories, that has been developed as a distinctive adaptive advantage. Actually, research suggests that it has developed to alleviate the selection pressure of information acquisition. Indeed, first-hand experience is potentially storytelling allows acquiring information "safely and efficiently". It has been proved that widely divergent societies practice some form of storytelling, even the most isolated ones. And finally, we can say that "narrative is a highly complex psychological process, depending for its operation upon the integration of numerous cognitive mechanisms [...] and exhibits a degree of complexity that is unlikely to have arisen by chance" [3]. Storytelling has been described as a "social action" because it requires a teller and an audience. This ability has been developed through the ages because it allows the "pooling of information": we know the present thanks to the shared experiences with the other individuals, and we know the past thanks to the transmission of stories from generation to generation. In fact, it is impossible for one individual to gather through personal experiences all the knowledge potentially useful for him to survive. It is important to note that stories are far more memorable than another kind of information. For example, it's easier to remember a fairy tale than a 20 -digit number, even if the tale is way more complex. This is because telling and understanding stories actually were essential to the survival in the environment of evolutionary adaptiveness [2]. In fact, it has been proven that "people naturally think narratively rather than argumentatively or paradigmatically". Also, "a substantial amount of information [4-6] stored and retrieved from memory is episodic [7,8]". Lastly, "watching, retrieving, or repeatwatching stories results in what Aristotle refers to as "proper pleasure", a catharsis [4]". Given these premises, "specific brands and products often play pivotal roles 


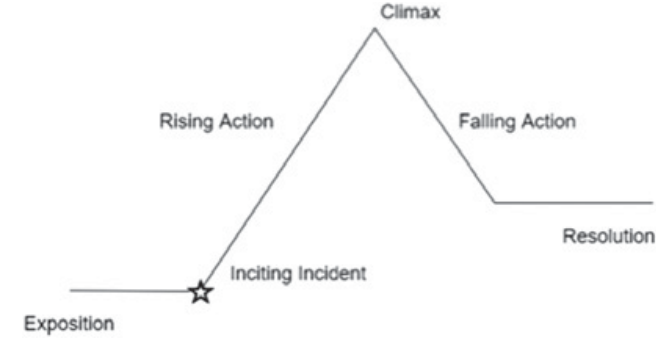

Figure 1. Piramyd of Freytag.

enabling consumers to achieve the proper pleasure that results in a consumer mentally and/or physically enacting a specific archetype - and reliving the experience by periodically retelling a given story". The brand-consumer storytelling and pleasure outcome builds on Bagozzi and Nataraajan's idea [9] that people need help in finding what makes them happy, and this is where marketing comes in" [10]. Nowadays, "storytelling" has been widely used as the definition of a content-based marketing technique. "All stories in storytelling can identify people and create emotional fellowship [11]. Studies on the development of a brand indicate the importance of developing a compelling story as one element of the brand icon $[10,12,13]$. These studies also argued that storytelling helps people get familiar with a product" [14]. A "consumer storytelling theory" has been developed during these years, to define a story's structure, which includes two important elements: chronology ("action occurs over time") and causality ("organized relationship between elements"). Furthermore, "a good story displays tension that includes one or more inciting incidents preceded by conditions or settings that initiate the unconscious/conscious identification of one or more goals, with actions by a protagonist and possibly additional actors resulting in an outcome; the temporary occurrences of world blocks (e.g., an antagonist temporarily preventing the protagonist from achieving the main goal) and/or personal blocks (e.g., the protagonist lacks the skill to perform an act necessary to reach the goal) serve to increase viewer and/or protagonist emotion and involvement in a story. Taking steps to overcome blocks such as seeking and gaining help from others occur frequently in stories" [10]. The above-mentioned structure refers to Gustav Freytag studies [15]. He developed a homonymous "pyramid", that offers a predictable and adaptable narrative scheme.

The storytelling-based communication strategy should be grounded for the most part $(80 \%)$ on content that uses emotion as a leverage. The remaining $20 \%$ of contents should refer to brand awareness or sales. For Food\&Wine companies, this technique is fundamental, because the emotional investment is insured by the nature itself of the products. In fact, because of the way agri-food products fit in interpersonal relations, they represent the fittest products for this kind of strategy. In the agri-food sector, the connection between consumer and brand is often based on associations between the product and its values. This bond can refer to: intrinsic organoleptic characteristics, to the product's status symbol and tribe symbol, the consumption patterns, the product's association with the country of origin and so forth. In conclusion, we can say that telling stories is a way to grant memorability [16].

\section{Storytelling literature in food and wine}

Currently, several papers evaluate the influence of storytelling practices on different foodstuffs. In 2006, Tellstrom et al. [17] have demonstrated that the producthomeland association is an effective way to draw consumers' attention for marketing consultants. Semistructured interview and "stimulus product" methods were used. It was found that Geographical Indication is considered an important commercial resource. Between the beneficial effects, authenticity, exoticism, tastefulness, manual production, etc. can be found [17]. Yong-sook Lee e Woo-jin shin in 2015 [18] explored the relevance of storytelling practices. Their study looks into the casestudy of a traditional sake brewery in Japan. The work has a qualitative approach and highlights the absence of previous studies on the topic of storytelling techniques applied to alcoholic beverages. Kida brewery, the subject of this study, has reinvented the sake industry with its strategy. Customers can visit the brewery after obtaining information on the website. They can touch, see and compare different kinds of rice and they can witness the process of polishing, fermentation and distillation. Lastly, there is the opportunity of tasting and brewing their own sake blend. A museum on the tradition and history of Kida brewery is present, too. The authors underline how storytelling must focus on emphasizing the emotional and tourism-related factor, such as history, process, ingredients, brand, culture and traditions [18]. In the paper "Delicious words", the researchers evaluated "the impact of short storytelling messages on consumer preferences for variations of a new processed meat product". "Product stimuli" method and choice experiment designs were used to prove that short storytelling messages may affect both interested and uninterested consumers. They have found that storytelling techniques can be successfully used in the launch on the market of new products variants and in broadening the spectrum of potential customers. the authors suggest creating appealing stories, using for that purpose intangible attributes like naturalness, authenticity and quality [19]. Lastly, Delgado-Ballester e Fernàndez-Sabiote in 2006 analyzed storytelling practices by Spanish brands from different sectors [20]. Through content analysis, they identified the differences between the companies' strategies. Characteristic elements of the stories were also discovered. Thanks to cluster analysis four different kinds of stories were identified. Results show that the fundamental elements of the stories are four (consistently with the pre-existing literature): authenticity, conciseness, reversal and humour, even if there were substantial differences between the brands [20]. When it comes to wine, it is important to mention studies that explore its relationship with emotions and storytelling. For example, a study carried on Australian consumers, proved how the information presented on wine back-labels or wine company websites “influences consumers' expected liking, informed liking, wine-evoked emotions and willingness to pay for Australian white wines. [...] Results showed that information level had a significant effect on all investigated variables [...] and a substantial increase in willingness to pay" [21]. Sillani et al. [22] also studied "the presence of variations in the reactions of different types of audiences to certain communication tools for wine". 
Different groups of Italian consumers were considered. From the conjoint analysis, the researchers discovered that "textual language was more effective with professionals, while the photographic language was more effective with tourists. Supplementary information on the organic production methods, in addition to the mandatory labelling requirements, increased the preferences of professionals and wine tourists and was counterproductive with the general customers [22]. Besides, Malorgio et al. in 2008 [23] state that, for Italian consumers, "the use of the designation of origin to assess quality at the moment of purchase requires a certain level of product knowledge and involvement" while the individual brand is described as a helpful tool to identify the wine and to link it to past consumption experiences. Therefore, "the brand represents a risk-reducing strategy for consumers". In fact, "The Designation of Origin lower influence, when compared to the variety or the brand, might derive from its lower "accessibility". Namely, occasional consumers seem to have difficulties in using this quality signal and prefer the brand attribute" [23,24]. It is essential to stress that "storytelling is what allows brand to become icons" [25].

\section{Wine and territory bond: One good story to tell}

Focusing on the wine's bond to its producing country, many studies have been made. Johnson and Bruwer [26] note that consumers rely on extrinsic cues such as brand name and origin when assessing product quality. In 2007 they investigated the effect of the wine regions included on the wine label on consumers' quality perception of the product. They also examined the effectiveness of this cue with respect to other information. The authors found that "almost without exception, the addition of regional information on a label increased consumer confidence in the quality of the product" [26]. Consistently, McCutcheon et al. [27] have come almost to the same conclusions for Australian domestic market. By administering an online survey to an extensive group of consumers, they discovered that "region of origin is an important choice factor in the wine-buying decision making process of consumers, but particularly so in terms of its multidimensional nature (region name, wine type and grape variety). The most important choice factors in consumers' wine-buying decisions are quality, price, grape variety, wine style and region of origin" [27]. In accordance, Martinez-Carrasco et al. found that for Spanish consumers' "ethnocentrism or geographical association is still a major consumption motivation when choosing a wine" [28]. Moreover Guidry's et al. research "shows the strong effect of origin even when participants actually sample the (identical) wines" [29]. In fact, 257 Texas university students rated the same wine differently, because one bottle was labelled as from Texas and the other as from France. "Proving that a wine's origin has a strong effect on consumers' preferences and price perceptions" [20].

\section{Social media and wine: The place to tell stories in the 2.0 society}

With the development of the Internet, social networks have changed and, in particular, updated the marketing strategies. One of the industries in which both Web 2.0 and social media have a strong impact is the wine industry [30]. Web 2.0 allows users to interact and the level of involvement of social media is the benchmark for online strategies. Web 2.0, therefore, changed the possibilities for wine marketing and consumers to become familiar with products. The Internet and social media, enable businesses to track actions and conversations on the web and, because of this, to have unique source of information and analysis. The key issue, nowadays, for marketing managers is how to make full use of social media for reaching marketing goals and support marketing strategies [31-33]. Marketing of social media from mid2000 gave new advertising opportunities, products and services. Consumers are spending an increasing amount of time on social media, brands have discovered their power marketing opportunity to attract consumers, encourage consumption and build relationships. Companies mostly use social media to announce, generate promotional contests, provide information, and customer services $[32,34]$. According to Flint et al. [31] countries show differences in the use of new media and, consequently, the companies located in these environments, are reflecting the higher level of adoption. According to Kaplan \& Haenlein [35] , general social channels include Facebook, Twitter, Instagram, Pinterest, Wikipedia, YouTube, TripAdvisor, Yelp and more, as well as many mobile applications for smartphones. As argued by Forbes et al. [36], social media platforms include those developed for social networking, text messaging, podcasts, wikis, blogs, photo sharing, streaming videos and online forums. These platforms are increasingly available to consumers (and businesses), including through mobile devices. The most well-known platforms include mechanisms for social or professional networking sites such as Facebook and LinkedIn, tools for sharing videos such as YouTube and blogging engines like Twitter. According to the data, as cited by Dolan et al. [37], there are currently more than 15 million marks in the world that have a company registered with Facebook. More than 70 percent of Internet users around the world are connected to the social networks, and further, more than 50 percent of social network users used to interact with brands on the social platforms. Numerous studies describe how social media can be useful to the wine consumers and wineries [34]. Thach \& Olsen [38] mention three different types of users of the wine: Low, Moderate and High Spenders. All wine users allocate more wine purchases to different channels, but it is interesting that all three user groups use wine apps and social media applications. However, the low spender uses wine apps to find coupons for grocery stores, whereas the Moderate and High Spenders use social media for information and, perhaps, to communicate with friends in their social media networks. Furthermore, it was found by Dean \& Forbes [36] that many winery websites and social media portals (e.g. Facebook) in the wine industry use those sites to promote sales, generate a buzz for the brand, and provide a home for their brand community. In the wine industry, manufacturers are most interested in consumers' opinion hink and what they say about their wines. Therefore, studies were also made in this direction. According to Flint et al. [31], third-party research firms can extract this information from sources such as blogs by opinion leaders, Facebook posts, tweets, forums and even Instagram posts. Just identifying whether comments 
are positive or negative in association with a brand is known as sentiment analysis. Today, research firms can go further and gain insights to what consumers are saying in detail. This may seem rather easy, to interpret what people are saying, but, given that one month's posts in only one of these online social media applications could amount to the equivalent of seven thousand pages of text if printed, you realize that special tools and skills are needed to mine those data. As noted, communication is changing and both consumer and buyer habits are becoming more dynamic. According to Flint et al. [31], new technologies have an impact on how companies communicate and open up new opportunities. The old communication approach is less credible in relation to the new media (media with an exceptional focus, very measurable feedback and tailored messages). New items in the communication process are becoming some strong message senders. Contemporary communication must deal, in a global competitive environment, with new technologies and their impact on behaviours (the number of new social media and new apps is growing: user-generated-content media allows users to gain control and power in communication, Big Data are now available, E-commerce). After research and detailed analysis of websites and online tools, we found that social networks allow users to use their accounts free, while they allow businesses additional payable advertising. With such advertising a company can reach the target audience with its messages, therefore, on the basis of their targets, it could choose users of social networks depending on their demographic characteristics, behaviour, interests and location. The advertising results can be measurable, since the social networks enable the analytic of all users' activities, in particular the target audience. This provides to the company complete control over the effectiveness of marketing strategies. According to Szolnoki et al. [40], social media are becoming increasingly important for obtaining and giving feedback on wine. According to Higgins et al. [41], they allow to the businesses to include consumers in a more individualized manner and provide for their special interests and accelerate awareness of their wine. A new wave of behaviour and technological change has, thus, caused a number of industries to modernise heir communications strategies. Various industries are increasingly recognizing the importance of social media and they try to use the tools of social media to reach both generation $\mathrm{Y}$ and baby boomers, to acquire new customers, retain existing ones and manage their reputations. The wine industry is also included in this trend, although slightly slower because of the culture industry, especially with the vast majority of small and medium-sized enterprises which are product/production oriented and invest primarily in winemaking or viticulture knowledge; there is conviction that marketing is time-consuming and expensive. Marketing experts are not yet considered as strategic in this industry by many, at least not compared to other consumer goods industries. In any case, a successful wine company must deal with communication decisions and cannot stop these new challenging situations [31,33] and have to accept advertising opportunities offered by new technologies. However, we can conclude that the increase of the social media uses and their rapid adoption of consumers and businesses represents, according to Wilson \& Quinton [33] a phenomenon of the Internet economy.

\section{Concluding remarks}

There is a growing perspective in wine marketing. Storytelling is now institutionalized as a communication approach to generate value mainly true the communication of values and emotions to consumers. Literature shows that emotional involvement, in fact, creates bonds between product/brand and customers. Such an approach tries to solve the enormous complexity of highly differentiated markets, such as wine, in which consumers have difficult time to create loyalty with the products or get informed in order to get emotional involvement from the consumption. The fundamental contribution to the development of those strategies is given by the social media, which are counting a growing number of adhesion from the side both consumers and companies. The Web 2.0 creates connection B2C while textual and visual contents speedup the emotional connections. After analyzing the issue, many questions arise. Whether the strategy could actually be long lasting and create a "perpetual effect", whether there is an actually gain in profit by adopting such a strategy, whether storytelling diminishes the importance of other strategies based on certifications and/or geographical indications. In order to do so, quantitative analysis on case studies of companies and their experiences is going to be welcome in the further literature contributions.

\section{References}

[1] T. Pencarelli, F. Forlani, V Congresso Internazionale Marketing Trends (2006), p. 1

[2] M.S. Sugiyama, Food, Evol. Human Behav. 22, 221 (2001)

[3] O.W. Williams, Pioneer Surveyor, Frontier Lawyer: the Personal Narrative of OW Williams (Texas Western College Press, 1877) (1966)

[4] A. Hiltunen, Aristotle in Hollywood: The Anatomy of Successful Storytelling (Intellect Books, 2002)

[5] A. McKee, Textual Analysis: A Beginner's Guide (Sage, 2003)

[6] K.E. Weick, Sensemaking in Organizations 3. Sage, (1995)

[7] S. Fournier, J. Consumer Res. 24, 343 (1998)

[8] R.C. Schank, Tell me a Story: A New Look at Real and Artificial Memory (Charles Scribner's Sons, 1990)

[9] R.P. Bagozzi, R. Nataraajan, Psychology Mark. 17, 1 (2000)

[10] A.G. Woodside, S. Sood, K.E. Miller, Psychology Mark. 25, 97 (2008)

[11] S. Herskovitz, M. Crystal, J. Business Strategy 31, 21 (2010)

[12] B.D.M. Boje, Acad. Manage. J. 38, 997 (1995)

[13] N.L. Holt, Int. J. Qualitative Methods 2, 18 (2003)

[14] Y. Lee, S. Woo-jin, Springer 9, 281 (2015)

[15] K. Wheeler, Freytag's Pyramid (2004)

[16] T. Cattivelli, Emozionare con lo Storytelling (2018)

[17] R. Tellstrom, I.-B. Gustafsson, L. Mossberg, Place Branding 2, 130 (2006)

[18] Y.S. Lee, W.J. Shin, Service Business 9, 281 (2015)

[19] M.H. Fenger, J. Aschemann-Witzel, F. Hansen, K.G. Gurgnet, Food Qual. Preference 41, 237 (2015)

[20] E. Delgado-Ballester, E. Fernandez-Sabiote, Spanish J. Mark. - ESIC 20, 115 (2006)

[21] L. Danner, et al., Food Res. Int. 99, 263 (2017) 
[22] S. Sillani, A. Miccoli, F. Nassivera, Wine Econ. Policy 6, 28 (2017)

[23] G. Malorgio, A. Hertzberg, C. Grazia, Italian Wine Consumer Behaviour and Wineries Responsive Capacity (Belgium, EAAE, 2008)

[24] A. Hertzberg, G. Malorgio, New Medit 4, 40 (2008)

[25] P. Mora, F. Livat, Wine Econ. Policy 2, 3 (2013)

[26] R. Johnson, J. Bruwer, Int. J. Wine Business Res. 19, 276 (2007)

[27] E. McCutcheon, J. Bruwer, E. Li, Int. J. Wine Business Res. 21, 212 (2009)

[28] L. Martinez-Carrasco, M. Brugarolas, A. Martinez-Poveda, J. Wine Res. 16, 213 (2005)

[29] J.A. Guidry, B.J. Babin, W.G. Graziano, J.W. Schneider, Int. J. Wine Business Res. 21, 298 (2009)

[30] T. Tomazic, J. Local Self-Government 15 (2017)

[31] D.J. Flint, P. Signori, S.L. Golicic, Contemporary Wine Marketing and Supply Chain Management, A Global Perspective (New York, Palgrave Macmillan, 2016)

[32] G. Tsimonis, S. Dimitriadis, Mark. Intell. Planning 32, 328 (2013)

[33] D. Wilson, S. Quinton, Int. J. Wine Business Res. 24, 271 (2012)

[34] L. Thach, T. Lease, M. Barton, J. Direct Data Digital Mark. Pract. 1 (2016)
[35] A.M. Kaplan, M. Haenlein, Business Horizons 53, 59 (2010)

[36] S. Forbes, S. Goodman, R. Dolan, J. New Business Ideas Trends 13, 1 (2015)

[37] R. Dolan, S. Goodman, C. Habel, Aust. New Zealand Grapegrower Winemaker 608, 13 (2014)

[38] L. Thach, J. Olsen, Wine Economics Policy 4, 53 (2015)

[39] D.L. Dean, S.L. Forbes, Successful Social Media and Ecommerce Strategies in the Wine Industry, edited by G. Szolnoki (Hampshire, Palgrave Macmillan, 2016), p. 115

[40] G. Szolnoki, L. Thach, D. Kolb, Current Status of Global Wine Ecommerce and Social Media, Successful Social Media and Ecommerce Strategies in the Wine Industry, edited by G. Szolnoki (Hampshire, Palgrave Macmillan, 2016), p. 1

[41] L.M. Higgins, M.M. Wolf, M.J. Wolf, Successful Social Media and Ecommerce Strategies in the Wine Industry, edited by G. Szolnoki (Hampshire, Palgrave Macmillan, 2016), p. 13

[42] Qualivita - Ismea, Comunicato stampa $\mathrm{n}^{\circ} 1$. s.l.:s.n. (2017)

[43] Rabobank International Food \& Agribusiness Research, Wine is business, shifting demand and distribution: major drivers reshaping the wine industry., Utrecht: s.n. (2003) 